\title{
Factors Associated With the Provision of Sexual Health Care by Nursing Students in Hospitals: An Analytical Cross-Sectional Study
}

\author{
Yen-Hui Wang ${ }^{1,2} \&$ Jiin-Ru Rong ${ }^{1}$ \\ ${ }^{1}$ Department of Nursing, National Taipei University of Nursing and Health Sciences, Taipei, Taiwan \\ ${ }^{2}$ Department of Nursing, Mackay Junior College of Medicine, Nursing and Management, Taipei, Taiwan \\ Correspondence: Prof. Jiin-Ru Rong, Department of Nursing, National Taipei University of Nursing and Health \\ Sciences, No.365, Ming-te Road, Poitou District, Taipei City, Taiwan. Tel: 886-2-2822-7101.
}

Received: July 11, 2019

Accepted: August 1, 2019

Online Published: August 23, 2019

doi:10.20849/ijsn.v4i3.622

URL: https://doi.org/10.20849/ijsn.v4i3.622

\begin{abstract}
This study examined the knowledge and experiences regarding the execution of sexual health care, as well as the sexual self-concepts, of nursing students and their effects on students' competency in providing sexual health care in general hospitals. This was a cross-sectional study in which data was collected from 428 nursing students attending nursing schools in Northern Taiwan. Data was gathered using a self-administered questionnaire which collected information on demographic characteristics, sexual self-concept, sexual health care knowledge, skills, and experiences. The results showed that an average of $81.2 \%$ of the items in the sexual health care knowledge and skills scale were answered correctly. Although the students' did not have sexual anxiety, they reported mild negative self-evaluations of their sexual consciousness, and uncertainty regarding sexual needs and activities. The variables of receiving sexual health care training, experiences of sexual harassment, sexual self-concept, knowledge of sexual health care, and experiences of the execution of sexual health care were able to effectively explain $66 \%$ of the variation in competency in providing sexual health care. Moreover, students' previous experiences of the execution of sexual health care $(\beta=.784, p<.0001)$ were found to have the strongest influence on their competency in providing sexual health care. This study showed that nursing students' competence in providing sexual health care in hospitals requires participation, engagement in, and the accumulation of practical care experience in order to develop sexual health care competencies and establish a positive caring attitude.
\end{abstract}

Keywords: sexual self-concept, knowledge of sexual health care, competencies of sexual health care

\section{Introduction}

The World Health Organization (WHO) has stressed that sexual health encompasses various aspects of health, including physiological, emotional, intellectual, social, and spiritual health, all of which contribute to the development of a person's self-identity and personality, and allow him or her to engage in positive communications and love-related interactions with the outside world (WHO, 2018). The WHO also indicated that every individual should engage in sexual behaviors, relations, and experiences that are positive, respectful, joyful, safe, non-coercive, non-discriminatory, and non-violent, and that emphasize safety in regard to sex and fertility. Even though sexual health care is an important field in nursing, nurses still find it difficult and uncomfortable to provide sexual health care, since they are bogged down by a complex variety of clinical matters and usually focus only on disease treatment and physical symptom management. Most nurses understand that diseases and treatments can affect a patient's sexual health, and that it is their duty to discuss sexual health-related issues (with their patients) and provide sex counseling (Saunamaki, Andersson, \& Engstrom, 2010; $\mathrm{Wu}$, Chien, Ruan, \& Hsu, 2013); however, sexual health care is often overlooked in the actual clinical environment, where sexual health assessments are rarely if ever performed (Sung, Yeh, \& Lin, 2010; Ho \& Fernández, 2006; Jaarsma et al., 2010). These trends have demonstrated the passivity and inadequacies of clinical nursing with respect to sexual health care, and thus, a further examination of this topic should be conducted, with the aim of identifying ways in which this situation can be improved.

As early as 1973, the WHO had already called for medical and nursing schools to provide their students with sexual health knowledge and information. In the 1980s, various professional societies in the US's nursing community began to add nursing assessments, diagnoses, and interventions for sexual health to their lists of standard nursing tasks, and to incorporate sexual function changes as a topic in their nursing education curricula. 
Thereafter, research on sexology, sexual function, and sexual health issues began to emerge worldwide, kick-starting the growth of sex-related knowledge, attitudes, education, and research in the field of nursing (Waterhouse, 1996). However, Taiwan's nursing education system has yet to emphasize and develop nursing courses pertaining to sexuality, sexual health, and sexual function, and there is also a lack of studies on these topics. As a result, nursing students and nurses who encounter sexual health-related problems are constrained by their lack of knowledge and skills, which prevents them from providing sexual health care and limits the coverage of their care (Lee, Tsai, Huang, \& Huang, 2017; Wu, et. al., 2013). Wu et al. (2013) examined the sexual health care perspectives (and relevant correlations) of 239 nurses and found that nurses with particular characteristics (i.e. older, educated to a higher level, possessing more years of nursing experience, and studied sex education) demonstrated a higher level of comfort and higher recognition of professional suitability. They also found that the nurses' proficiency and confidence, with regard to their ability to provide sexual health care, were significantly correlated to their practical work experience with sexual health care and whether they had attended sexual health care-related courses. A study by Tsai and Hsiung (2001) indicated that the in-service training nurses from a nursing department were unwilling to inquire about their patients' sexual history due to factors related to the nurses themselves, their patients, and the environment. The nurse-related factors included personal embarrassment, the lack of relevant knowledge, the fear of infringing patient privacy, and the lack practical ability. The patient-related factors included the patients' refusal, the patients' lack of such needs, and the patients' attitudes. The environmental factors included the department's position that it was not necessary to provide such a form of care, and the lack of privacy in the environment. These findings have shown that the nursing education in Taiwan has yet to integrate sexual health care education, and that educational factors such as the lack of sexual health care courses have affected the knowledge, attitudes, and skills of nursing students with respect to the implementation of sexual health care.

In both local and overseas studies on the relationship between the sexual knowledge and attitudes of nurses and their nursing behavior, it was found that most nurses would only engage in discussions with patients who broached the subject of sexual problems, and that few nurses were actively assessing the sexual needs of their patients and providing sexual health guidance (Tsai, \& Hsiung, 2001). These findings indicate that we should work on improving health education relating to nurses' handling of patients' sexual problems. These studies also revealed that the main factors contributing to the sexual health care-related problems faced by nurses and nursing students included the lack of sexual health care knowledge, skills, and experience, and the lack of sexual health care education and training; these factors often led nursing students and professionals into awkward situations when they were discussing sexual health issues, and also prevented them from actively conducting sexual health care activities (Bartlik, Rosenfeld, \& Beaton, 2005; Bikker, Fitzpatrick, Murphy, Forster, \& Mercer, 2017; Wu, et. al., 2013). When providing sexual health care, nurses often encounter the following problems: 1. difficulties in tracking or evaluating the effectiveness of sexual health education; 2. difficulties in conducting in-depth discussions without prior establishment of trust; 3 . lack of appropriate sexual health information and teaching materials; 4. lack of quiet environments that offer privacy; and 5. the overly broad and complex nature of sexual health education that prevents one from completing said education independently. When assessing the sexual health history of their patients, nursing students face the following hindering factors: 1 . personal lack of knowledge and understanding with respect to sex-related issues, leading to concerns that one is unable to provide a patient with the right recommendations and objective guidance and, consequently, creating a situation in which the patient's sexual history is avoided or ignored in evaluations; 2. concerns over the possible triggering of emotional responses in oneself and a patient when discussing such issues (e.g. a nurse might be concerned that he or she will be perceived by the patient to be someone who is interested in these issues); and 3. inability to utilize the appropriate words, concerns over the possibility that a patient will not understand the medical terms being used, and concerns over the possibility of sounding too vulgar when resorting to the use of slang (Tsai, \& Hsiung, 2001; Lee, Tsai, Huang, \& Huang, 2017).

Past studies have shown that nurses who attended sexology courses demonstrated a significant positive change in their attitudes toward sexual health care (Sung \& Lin, 2013), and that those who studied sexlogy-related education demonstrated a higher level of comfort with respect to the sexual counseling process (Sung, Yeh, \& Lin, 2010). Taiwan's nursing education focuses primarily on nursing topics relating to diseases and treatments, as well as physiology, disease, or treatment-oriented health guidance for patients and their families, while neglecting interventional measures relating to sexual health care (Lee, 2015; Wu, et. al., 2013; Tsai, \& Hsiung, 2001; Chuang, \& Chin, 2003). These findings could be linked to the limited range of sexual health care courses and teaching materials offered by the local nursing education system, as well as the lack of sexual health knowledge and clinical experience among nurses, such that their ability to provide sexual health care as a nursing service is affected (Tsai, \& Hsiung, 2001; Chuang, \& Chin, 2003). 
Furthermore, a nurse's physical and mental development and experience in relation to sexual health will influence his or her attitude toward the sexual health care needs of patients. Nursing students at the tertiary level are still in the adolescence and early adulthood phase of their development, and their physical and mental health in relation to sexuality are still developing. Thus, their sexual self-concept and sexual health will influence how they learn nursing practices pertaining to sexual health care. Since no studies to date have performed an in-depth exploration of this topic, our study aimed to explore the factors that influence the practical ability of nursing students with respect to sexual health care, so as to lay a foundation for improving their ability in this area.

\section{Method}

In this cross-sectional correlational study, convenience sampling was utilized to recruit nursing students from the college of nursing of a university in northern Taiwan as research participants. After providing consent to participate in the questionnaire survey and signing the informed consent for the study, a student was considered a research participant and, subsequently, assessed to determine his or her current knowledge, attitude, and skills with respect to the provision of sexual health care.

\subsection{Research Participants}

A convenience sampling of nursing students (comprising mostly students from a college of nursing in northern Taiwan) was obtained. 428 nursing students who met the above requirements and were willing to participate in the study were eventually included in the research sample.

\subsection{Research Tools}

The study was a cross-sectional correlational study that utilized a structured questionnaire for data collection. The various assessment tools are described below.

\subsubsection{Basic Information Questionnaire for Research Participants}

Based on the relevant literature, this study examined the demographic characteristics of age, gender, marital status, intimate partner, sexual harassment, and experience with practical work relating to sexual health care.

\subsubsection{Sexual Self-concept Scale}

The Multidimensional Sexual Self-Concept Questionnaire (MSSCQ) proposed by Snell (1996) was utilized, and after referring to past studies that explored the psychological and social conditions of university students aged 16 to 25 years, the five following variables of the scale were selected as measurement tools for measuring the sexual self-concept of university nursing students: sexual anxiety, sexual self-efficacy, sexual consciousness, sexual monitoring and awareness, and sexual self-esteem.

Lin (2004) used a Chinese version of the MSSCQ to examine psychiatric nurses in Taiwan $(\mathrm{N}=163)$ and found that the five sub-scales, namely sexual anxiety, sexual self-efficacy, sexual consciousness, sexual self-esteem, and sexual monitoring and awareness, had Cronbach's $\alpha$ values of $.84, .85, .78, .91$, and .89 , respectively. In that study, five-point Likert items were used, with a higher score indicating a more positive sexual self-concept.

\subsubsection{Sexual Health Care Knowledge Scale}

The tool, which contained 57 items, was developed from Lin's (2009) empirical research results and past studies of sexual health. This sexual health care knowledge and attitude scale consisted of four main sections (with a total of 57 items): 1. basic concepts of sexology and fertility; 2. basic concepts of sexually transmitted diseases and related treatments; 3. sexual dysfunction and related treatments; and 4. knowledge relating to sexual health care practices. Three response options were available for each item, namely "Agree," "Disagree," and "Not sure"; the questionnaire was self-administered to the nursing students and scored between 0 and 57. The Cronbach's $\alpha$ value for the nursing students' self-evaluation was .73.

\subsubsection{Sexual Health Care Skills Scale}

The scale was used primarily to understand the nursing students' practical experiences and competency with respect to the provision of sexual health care to adult patients in hospitals. In this study, the sexual health care skills scale, which includes two dimensions, was used to measure the students' sexual health care experiences. One dimension was used to evaluate their experiences with the execution of clinical sexual health care, while the other dimension was used to assess their competence in providing sexual health care in hospitals. The sexual health care skills scale is a self-reported questionnaire that covers five areas of sexual health care clinical skills and was developed based on empirical studies of sexual health care (Lin, 2009). The five areas are as follows: 1. the evaluation of general health functions and status (9 items); the Cronbach's $\alpha$ for this study was .86 . 2. The evaluation of sexual development and sexual health issues (15 items); the Cronbach's $\alpha$ for this study was .92. 3 . 
The execution of basic sexual health care practices ( 8 items); the Cronbach's $\alpha$ for this study was .87 .4 . The maintenance of patients' sexual well-being during hospitalization (7 items); the Cronbach's $\alpha$ for this study was .91. 5. The implementation of nursing for sexually transmitted diseases ( 8 items); the Cronbach's $\alpha$ for this study was .93. In this scale, a five-point Likert scale is used, with the lowest score meaning that the respondent does not have experience in providing sexual health care in hospitals. Otherwise, lower scores regarding competency in sexual health care execution skills indicate that the respondent does not have confidence in providing sexual health care to patients. The two dimensions of sexual health care skills (i.e. care experience, execution competence) were each assigned scores between 47 and 235 points, with higher scores indicating more experience or competence in providing sexual health care than lower scores.

\subsection{Data Collection and Procedures}

The Mackay Memorial Hospital Institutional Review Board (18MMHIS131e) approved this study. Informed consent was obtained at the time of recruitment. The participants consisted primarily of nursing students from a college of nursing and were recruited through their classes. The students were briefed on the research objectives and procedures and given an anonymous questionnaire to complete (the students could opt to participate or not to participate in the questionnaire session), and under conditions observable by the researchers, the questionnaire was filled in and collected.

\subsection{Data Analysis}

Of the 434 participants who were initially recruited, six of them submitted questionnaires with 20 or more unanswered items. Due to the excessive number of missing values (more than $20 \%$ of the questionnaire) associated with these six participants, their data were removed, resulting in a sample of 428 participants being officially used in the study's data analysis. Based on the study's research objectives, a descriptive statistical analysis was performed to examine the nursing students' demographic variables, as well as their performance for other variables. The student's t-test and one-way ANOVA were utilized to test for inter-group differences pertaining to the nursing students' demographic variables. Pearson's correlation was utilized to test for correlations associated with each variable, and hierarchical multiple regression was performed to verify the influence of the nursing students' demographic variables and sexual self-concept on their sexual health care knowledge and sexual health care practical skills (i.e. experience, execution proficiency).

\subsection{Ethical Considerations}

The recruitment of participants began only after the study was reviewed and approved by the relevant research ethics committee. The participants consisted primarily of nursing students from a college of nursing and were recruited through their classes. The students were briefed on the research objectives and procedures and given an anonymous questionnaire to complete only after they had consented in writing to participating in the study in writing. Subsequently, the questionnaire was filled in and collected. A participant could opt to leave the study at any point while filling in the questionnaire, and during the briefing that the participants received, it was strongly stressed that their rights as students would not be compromised as a result of their participation in this study. As for the participating student's data, the researchers undertook the responsibility of maintaining their confidentiality.

\section{Results}

\subsection{Demographic Characteristics}

Among the 428 participants recruited for this study, the average age was 22.61 years $(\mathrm{SD}=4.06)$, and apart from 12 male students $(2.4 \%)$, the rest were female students. $96 \%$ of the participants were unmarried (the remaining $4 \%$ were all married); however, nearly $60 \%$ of the students already had a partner. Furthermore, $70 \%$ of the students had no experience of being sexual harassed, while $28.5 \%$ indicated that they had experienced light to moderate levels of sexual harassment. More than $70 \%$ of them had yet to receive sexual health care training (see Table 1). 
Table 1. Demographics of subjects $(\mathrm{N}=428)$

\begin{tabular}{lcc}
\hline Variables & $n$ & $\%$ \\
\hline Gender & 12 & 2.4 \\
\hline Male & 416 & 97.6 \\
\hline Female & & \\
\hline Marital Status & 411 & 96.0 \\
\hline Unmarried & 13 & 3.0 \\
\hline Married & 4 & .9 \\
\hline Cohabitating & & \\
\hline Intimate Partner & 245 & 57.2 \\
\hline No & 183 & 42.8 \\
\hline$\quad$ Yes & 301 & \\
\hline Sexual Harassment & 101 & 70.3 \\
\hline None & 21 & 23.6 \\
\hline Light degree & 5 & 4.9 \\
\hline Moderate degree & & 5 \\
\hline Serious degree & 304 & 71.0 \\
\hline Received Sexual Health Care Training & 124 & 29.0 \\
\hline Never & & \\
\hline Yes & & \\
\hline
\end{tabular}

\subsection{Sexual Self-concept of Nursing Students}

With respect to the five sexual self-concept indicators, first, the students had no obvious sexual anxiety. However, the students self-evaluated themselves as being at moderate levels (for sexual consciousness and sexual self-efficacy) to low levels (for sexual self-esteem and sexual monitoring) in terms of the other four sexual self-concepts. That is, the students had relatively negative self-evaluations regarding those four sexual self-concepts (see Table 2).

Table 2. Description of sexual self-concepts $(N=428)$

\begin{tabular}{lccccc}
\hline \multicolumn{1}{c}{ Variables } & Mean & $S D$ & Minimum & Maximum & Range \\
\hline Sexual anxiety & 21.41 & 3.39 & 5 & 25 & $5-25$ \\
\hline Sexual self-efficacy & 15.89 & 5.19 & 5 & 25 & $5-25$ \\
\hline Sexual consciousness & 17.19 & 4.58 & 5 & 25 & $5-25$ \\
\hline Sexual monitoring* & 12.89 & 4.26 & 5 & 25 & $5-25$ \\
\hline Sexual self-esteem* & 14.23 & 4.67 & 5 & 25 & $5-25$ \\
\hline Total Score & 81.56 & 16.56 & 40 & 123 & $25-125$ \\
\hline
\end{tabular}

Note: * indicates a score of less than 15 points

\subsection{Sexual Health Care Knowledge and Practical Skills}

\subsubsection{Sexual Health Care Knowledge}

In this study, the students had an average total score of $46.30(\mathrm{SD}=5.1)$, or $81.2 \%$, for the the sexual health care questions (range 0-57). This result indicated that the nursing students had adequate knowledge of sexual health. However, half of the students answered four questions regarding knowledge of fertility, sexually transmitted diseases, sexual health care strategies, and sexual dysfunction incorrectly.

\subsubsection{Practical Skills of Sexual Health Care}

In this study, two dimensions were used to evaluate the students' sexual health care experiences; one was used to evaluate their experiences with the execution of clinical sexual health care, while the other was used to assess 
their competency in providing sexual health care in hospitals. The results are described in the following sections.

\subsubsection{Experiences With the Execution of Clinical Sexual Health Care}

For the nursing students' practical work experience with respect to sexual health care, the total average score was $149.1(\mathrm{SD}=29.6)$ points, with an average of 3 points for each item. This finding indicated that they possessed a general level of experience in this area. Of the 47 items, a relatively low experience level (lower than 3 points) with respect to practical nursing work was reflected for 24 items. These items corresponded to the following dimensions: evaluation of sexual beliefs, evaluation of sexual health issues and sexual dysfunction, sexual health counseling and guidance, maintenance of sexual health and well-being of hospitalized patients, and management of sexual harassment and violence in wards.

\subsubsection{Competency in Providing Sexual Health Care in Hospitals}

For the nursing students' proficiency levels with respect to the execution of sexual health care-related practical work, the total average score was $143.2(\mathrm{SD}=29.8)$ points, with an average of 3 points for each item. This finding indicated that they possessed a moderate level of proficiency. The participants' infrequent execution of sexual health care work was reflected in their proficiency levels.

\subsection{Factors Influencing the Competency to Provide Sexual Health Care}

The results in Table 3 indicate that $66 \%$ of the variance in the competency in providing sexual health care in hospitals was explained by receiving sexual health care training, experiences of sexual harassment, sexual self-concept, knowledge of sexual health care, and experiences with the execution of sexual health care $(\mathrm{F}=166.35, \mathrm{p}<.0001)$. In addition, the regression coefficient of experiences with sexual health care was significantly different from zero $(\beta=.84, \mathrm{t}=26.60, \mathrm{p}<.0001)$. These results indicated that the students' experiences with the execution of sexual health care had direct effects on their competence in providing sexual health care. Moreover, the students' sexual self-concepts had a positive influence on their competence in providing sexual health care $(\beta=.73, \mathrm{t}=2.47, \mathrm{p}<.05)$, with the results showing that the students who had more positive sexual self-concepts had more confidence in the execution of sexual health care for patients. As shown in Table 3, the finding of no multicollinearity was further supported by the fact that the VIF and tolerance values were close to one.

Table 3. Regression analysis of factors influencing competency in providing sexual health care in hospitals $(N=428)$

\begin{tabular}{lllllll}
\hline \multicolumn{1}{c}{ Variables } & B & SE B & $\beta$ & Adj. R2 & Tolerance & VIF \\
\hline 1. Received Sexual Health Care Training & 3.167 & 1.874 & .043 & .983 & 1.018 \\
\hline 2. Experiences of Sexual Harassment & .629 & 1.339 & .013 & .983 & 1.017 \\
\hline 3. Sexual Self-concept & .132 & .053 & $.073^{*}$ & .912 & 1.096 \\
\hline 4. Knowledge of Sexual Health Care & -.111 & .170 & -.019 & .937 & 1.068 \\
\hline $\begin{array}{l}\text { 5. Experiences with the Execution of Sexual } \\
\text { Health Care }\end{array}$ & .784 & .029 & $.789^{* * *}$ & .908 & 1.101 \\
\hline Total R2 & & & & $.66^{* * *}$ & & \\
\hline
\end{tabular}

Note. $\Delta \mathrm{R} 2=.66(\mathrm{p}<.0001),{ }^{*} \mathrm{p}<.05, * * \mathrm{p}<.01, * * * \mathrm{p}<.001$

\section{Discussion}

Based on the research objectives and research hypothesis, the results and findings of this study are broken down and presented in the following three sections.

\subsection{Nursing Students'Sexual Self-concepts}

Among the participants in this study, $4 \%$ were married and $42.8 \%$ had intimate partners. These participants' sexual self-concept results were compared with those from a study by Su (2006), which examined the sexual self-concept and sexual health behavior of medical workers. The comparison indicated that the participants' had comparatively negative self-evaluations of various sexual self-concepts such as sexual self-efficacy, sexual consciousness, sexual self-monitoring, and sexual self-esteem. The participants were unsure of their own sexual 
needs or desires, did not pay attention to how other people react to their sex lives, and were not concerned about how their sexual beliefs and behaviors are evaluated by other people. In addition, the participants also did not exhibit sexual anxiety. Su's (2006) study revealed that adults who were married and had sexual experiences and fixed sexual partners had more positive sexual self-concept evaluations. As for the influence of marriage and sexual experience on the participants' sexual self-concepts, as well as their sexual health care knowledge-related attitudes and skills, these are topics that future studies could explore.

The participants were mostly students in early adulthood who were unmarried or otherwise lacked sexual experience; hence, they were less prone to discussing beliefs, opinions, behaviors, and other topics relating to sex. Many studies have shown that a positive sexual self-concept is an important key to maintaining and promoting sexual health, and our study's results have also indicated that the participants did not fully understand their sexual needs and lacked confidence in regard to their ability to and methods for handling their sexual needs. These findings not only affect the participants' sexual health, but may also affect their ability to identify, assess, and handle the sexual needs (and other relevant problems) of their patients. For these reasons, it is recommended that university nursing students first establish positive sexual self-concepts, such that they can take note of sexual health topics, discuss these topics with other people, and develop practical skills for sexual health care.

\subsection{Factors Influencing Nursing Students' Knowledge Relating to Sexual Health Care}

Sexual health care knowledge was evaluated using 57 items that assessed the accuracy of the participants' knowledge with respect to sexual health. For 35 of the sexual health knowledge items, the correct answer rate reached $80 \%$. The items that the participants answered incorrectly were as follows: Item 7 "Generally, if a man's sperm does not enter a woman's vagina, the woman cannot get pregnant"; Item 18 "Antibiotics are the main treatment for genital herpes"; Item 28 "When caring for an HIV positive patient, a nurse should proactively contact and provide health guidance to the patient's family or sexual partner(s)"; and Item 30 "Urinary system diseases are the main cause of sexual dysfunction." These findings indicated that there was a need for the participants to strengthen their sexual health care knowledge in regard to topics such as fertility, sexually transmitted diseases, health guidance, and the causes of sexual dysfunction.

Looking at the participants' knowledge relating to sexual health care knowledge, the most significant influencing factors were sexual self-concept and whether the participants had attended sex-related nursing courses. In a study involving 95 nursing students, Sung (2011) examined the effects of courses covering sexual issues and nursing professional growth, and found that the experimental group significantly outperformed the control group in terms of their sexual health care knowledge, sexual health care attitudes, and sexual health care self-efficacy. Bell and Bray (2014) conducted a qualitative study that examined a UK university's $(n=117)$ implementation of courses covering sexual health and sexually transmitted diseases, and found that students who were subjected to the course intervention experienced a significant improvement in their sexual health knowledge and attitudes and became more active in providing sexual health care. These findings were consistent with those from our study.

\subsection{Practical Ability and Influencing Factors Relating to Nursing Students' Provision of Sexual Health Care}

With respect to the participants' sexual health care (practical work) scores for common experiences and proficiency, they achieved an average proficiency score of 3.05 points, indicating that the participants had a moderate level of proficiency. However, this result highlighted the need for the participants to improve their proficiency levels with regard to the three aspects of work, namely the evaluation of sexual development and sexual health issues, the maintenance of patients' sexual well-being during hospitalization, and the implementation of nursing for sexually transmitted diseases.

Five variables (i.e. receiving sexual health care training, experiences of sexual harassment, sexual self-concept, knowledge of sexual health care, and experiences with the execution of sexual health care) were able to effectively explain $66 \%$ of the variation in the competence in providing sexual health care. In particular, experiences with the execution of sexual health care played a highly important role in influencing the competency in providing sexual health care in hospitals.

The above results have shown that the participants' proficiency and confidence with respect to sexual health care work, were significantly correlated to the sexual health care-related work experience that they acquired during clinical practice and the sex-related courses that they have attended. This indicated that the participants have to engage in practical activities the encompass observation, participation, and execution, so as to accumulate the experience that will help them to develop their sexual health care knowledge and a positive care attitude. With these considerations in mind, class-based and clinical learning relating to sexology could be implemented as an important teaching strategy. In a related local study, Sung \& Lin (2013) pointed out that practical sexual health or sexual health care courses differ from general memory-based or technical courses, since the development of 
sexual health knowledge will necessarily involve the establishment of concepts and the clarification of attitudes and values, as well as the development of a diverse range of teaching activities, the utilization of scenario-based discussions and drills, and the sharing of differing opinions. Rosen, Kountz, Zwicker, Leiblum, \& Wiegel (2006) examined a workshop attended by medical students from the Robert Wood Johnson Medical School; unlike the other courses generally offered by medical schools, the workshop incorporated sexual health-related educational and training courses that covered areas such as sexual dysfunction in women, the definitions of sexual issues, the taking of a sexual health history, the evaluation and diagnosis of sexual health issues, referrals for sexual health issues, the handling of legal ethics pertaining to sexual health issues, and sexual health issue management planning. Questionnaires were administered before and after the workshop to evaluate the workshop's impact on the medical students, in terms of their skills in managing sexual health-related issues faced by patients in various medical scenarios; their ability to provide medical care to patients and their self-evaluation of said ability; their perception regarding the importance of addressing patients' sexual health issues, and the frequency with which patients' sexual health issues are addressed. The results of that study revealed that there was a significant improvement in the students' knowledge, skills, and practical ability with respect to the sexual health issues faced by patients, and highlighted the importance of developing professional sexual health care skills and conducting sexual health care courses.

\section{Conclusion}

Limited research has examined the role of sexual self-concepts on the use of contraception or sexual health care competences. The significant results of this study showed that for the five sexual self-concept indicators, the students had no obvious sexual anxiety. However, the students self-evaluated themselves as being at moderate levels to low levels in terms of the other four sexual self-concepts of sexual consciousness, sexual self-esteem, sexual monitoring, and sexual self-efficacy. This result similar to the previous study, which indicates that young persons' experiences of sexual violence and low self-esteem were significantly related to ineffective use of contraception (Hensel, et. al., 2011; Nelson, et. al., 2017). Therefore, studies suggest that health professionals should provide Interventions to empower young people to recognize the effects of sexual violence and low self-esteem on the ability of sexual health care activity and have the skill of using safe contraception methods for caring sexual health (Nelson, et. al., 2017). Moreover, the sexual self-concept and experiences with the execution of sexual health care were the two most significant variables to predict the variation in the competence of providing sexual health care. Therefore, nursing students need to receive sexual health care education and training, to enhance the students ' sexual health care experiences, for empowering confidence in providing sexual health care for themselves and others.

Although other research has examined the role of contraceptive knowledge and found that knowledge influences contraceptive use of adolescents. The findings of this study showed that the experiences with the execution of sexual health care are the most significant predictor variable to the competences and skills of sexual health care. However, sexual health care knowledge cannot significantly predict the competences and skills of sexual health care. Therefore, the health profession should emphasize the holistic competence of safe sexual health care of young people, which should include knowledge, behavior skills, and attitude of sexual health care.

Also, in the prediction variables of competency in providing sexual health care, sexual self-concept, and sexual health care work experience showed a significant correlation. For these reasons, it is recommended that university nursing students first establish positive sexual self-concepts, and develop practical skills for sexual health care. Nursing students have to engage in practical activities of sexual health care the encompass observation, participation, and execution, to accumulate the experience that will help them to develop their sexual health care knowledge and a positive care attitude. In clinical practice courses, sexual health care should be implemented in the care of patients. Teachers should guide students to participate in sexual health-related assessments and implement nursing interventions to increase students' sexual health care experience in clinical care and thus improve their competence in providing sexual health care.

\section{Limitations and Suggestions}

\subsection{Limitations}

The study's limitations are as follows:

(1) The study was a cross-sectional study, in which a regression analysis was performed using only the research framework in order to understand inter-variable relationships and effects. It was not possible to examine the mutual and causal relationships between sexual self-concept and the knowledge of and practical work relating to sexual health care. 
(2) This study involved a large number of questionnaire items, which might have caused questionnaire fatigue and contributed to errors in the research results. In future studies, the number of questionnaire items can be reduced in order to avoid this problem.

\subsection{Suggestions}

Based on the research results, we propose the following suggestions for future research and nursing education:

(1) Future studies can expand their samples to include all nursing staff who are providing care to hospitalized adult patients, so as to better understand the scope of practical work and the content of work tasks with respect to sexual health care

(2) Nursing education should consider educational and training activities that cover sexual health care, and incorporate them into the educational curriculum for nursing students. Practical drills and training should also be implemented to raise the proficiency of nursing staff in performing the job scope for sexual health care.

\section{Acknowledgements}

We would like to express our gratitude to all of the 428 nursing college students from a university in northern Taiwan who kindly served as the research participants for this study. We would also like to thank Prof. Rong Jiin-Ru for her guidance and leadership with respect to the execution of the research methods, sample recruitment, and data analysis, without which the research would not have been completed and published.

\section{Funding}

This research did not receive any specific grant from funding agencies in the public, commercial or not-for-profit sectors.

\section{Conflicting Interests}

None

\section{References}

Bartlik, B. D., Rosenfeld, S., \& Beaton, C. (2005). Assessment of sexual function: Sexual history taking for health care practitioners. Epilepsy \& Behavior, 7, S15-S21. https://doi.org/10.1016/j.yebeh.2005.08.027

Bell, A., \& Bray, L. (2014). The knowledge and attitudes of student nurses towards patients with sexually transmitted infections: exploring changes to the curriculum. Nurse Education in Practice, 14(5), 512-517. https://doi.org/10.1016/j.nepr.2014.05.002.

Bikker, A. P., Fitzpatrick, B., Murphy, D., Forster, L., \& Mercer, S. W. (2017). Assessing the Consultation and Relational Empathy (CARE) Measure in sexual health nurses 'consultations. BioMed Central Nursing, 16(71), 1-9. https://doi.org/10.1186/s12912-017-0265-8

Chuang, W. L., \& Chin, C. C. (2003). Discussing sexual problems with myocardial infarction patients. The Journal of Nursing, 50(5), 69-73. https://doi.org/10.6224/JN.50.5.69.

Hensel, D. J., Fortenberry, J. D., O'Sullivan, L. F., \& Orr, D. P. (2011). The Developmental Association of Sexual Self-Concept with Sexual Behavior among Adolescent Women. Journal of Adolescence, 34(4), 675-684. https://doi.org/10.1016/j.adolescence.2010.09.005.

Ho, T. M., \& Fernandez, M. (2006). Patient's sexual health do we care enough?. Journal of Renal Care, 32(4), 183-186. Retrieved from https://www.ncbi.nlm.nih.gov/pubmed/?term=17345975

Jaarsma, T., Stomberg, A., Fridlund, B., De Geest, S., Martensson, J., Moons, P., et al.. (2010). Sexual counseling of cardiac patients: Nurse' perception of practice, responsibility and confidence. European Journal of Cardiovascular Nursing, 9(1), 24-29. https://doi.org/ 10.1016/j.ejcnurse.2009.11.003.

Lee, C. H., Tsai, L. Y., Huang, C. Y., \& Huang, Y. L. (2017). Sexual health care in disease progression. Journal of Taiwan Nurse Practitioners, 3(1), 70-77.

Lee, J. T. (2015). Bringing Gender Sensitivity into Sexual Health Care for Women with Gynecological Cancer. The Journal of Nursing, 62(1), 16-21. https://doi.org/10.6224/JN.62.1.16

Lin, M. C. (2009). Exploring nurses' practical experiences in providing sexual health care to acute and chronic illness patients. Unpublished master's thesis, National Taipei University of Nursing and Health Science, Taipei, Taiwan. Retrieved from https://hdl.handle.net/11296/zwg32t

Lin, Y. Y. (2004). An empirical study on attitudinal assessment instrument in chinese-based sexual self-concept on the MSSCQ. Unpublished master's thesis, National Taipei University of Nursing and Health Science, 
Taipei, Taiwan. Retrieved from https://hdl.handle.net/11296/2863ye

Nelson, D. B., Zhao, H., Corrado, R., Mastrogiannnis, D. M., \& Lepore, S. J. (2017). Preventing Unintended Pregnancy Among Young Sexually Active Women: Recognizing the Role of Violence, Self-Esteem, and Depressive Symptoms on Use of Contraception. Journal of Women's Health, 26(4), 352-360. https://doi.org/10.1089/jwh.2016.5753

Rosen, R., Kountz, D., Zwicker, T. P., Leiblum, S., \& Wiegel, M. (2006). Sexual communication skills in residency training: the Robert Wood Johnson model. International Society for Sexual Medicine, 3, 37-46. https://doi.org/10.1111/j.1743-6109.2005.00135.x

Saunamaki, N., Andersson, M., \& Engstrom, M. (2010). Discussing sexuality with patients: nurses' attitudes and beliefs. Journal of Advanced Nursing, 66(6), 1308-1316. https://doi.org/10.1111/j.1365-2648.2010.05260.x

Snell, W. E. Jr. (1996). The extended multidimentional sexuality questionnaire: Measuring psychological tendencies associated with human sexuality. Paper presented at the annual meeting of the Southwestern psychological Association, Houston, TX.

$\mathrm{Su}$, C. F. (2006). Effects of sexual self-concepts on sexual health behaviors. Unpublished master's thesis, National Taipei University of Nursing and Health Science, Taipei, Taiwan. Retrieved from https://hdl.handle.net/11296/fj7sws

Sung, S. C. (2011). The effectiveness of Sexual Issues and Nursing Profession Development Program in nursing students' knowledge, attitudes, and self-efficacy on sexual healthcare. Unpublished doctoral dissertation, Shu-Te University, Kaohsiung, Taiwan. https://doi.org/10.6829/STU.2011.00021

Sung, S. C., \& Lin, Y. C. (2013). Effectiveness of the sexual healthcare education in nursing students' knowledge, attitude,and self-efficacy on sexual healthcare. Nurse Education Today, 33(5), 498-503. https://doi.org/10.1016/j.nedt.2012.06.019

Sung, S. C., Yeh, M. Y., \& Lin, Y. C. (2010). An exploration of the nurses' perspectives and their current practice on sex consultation. Formosan Journal of Sexology, 16(1), 1-16. https://doi.org/ 10.6784/FJS.201004.0001

Tsai, L. Y. (2014). A study of nursing students' learning needs for patients' sexual health care. Unpublished doctoral dissertation, Chung Shan Medical University, New Taipei City, Taiwan. https://doi.org/10.6834/CSMU.2014.00044

Tsai, Y. F., \& Hsiung, P. C. (2001). Re-entry nursing student attitudes and behaviors in patient sexual history taking. Journal of Medical Education, 5(1), 33-39. https://doi.org/10.6145/jme

Waterhouse, J. (1996). Nursing practice related to sexuality: A review and recommendation. Journal of Research in Nursing, 1(6), 412-418. https://doi.org/10.1177/174498719600100603

World Health Organization (WHO). (2018). Defining sexual health. Retrieved from https://www.who.int/reproductivehealth/topics/sexual_health/sh_definitions/en/

Wu, H. P., Chien, S. C., Ruan, F. F., \& Hsu, H. Y. (2013). A study of sexual health care for patients provided by nurses in Kaohsiung. The Kaohsiung Journal of Nursing, 30(1), 21-36. https://doi.org/10.6692/KJN-2013-30-1-3

Yen, H. W., Liu, H. H., \& Ferng, J. Y. (2009). Influential factors in internet friend-making, dating and premarital sexual behaviors of adolescences. Taiwan Journal of Public Health, 28(4), 322-333. https://doi.org/10.6224/JN.56.2.53

\section{Copyrights}

Copyright for this article is retained by the author(s), with first publication rights granted to the journal.

This is an open-access article distributed under the terms and conditions of the Creative Commons Attribution license (http://creativecommons.org/licenses/by/4.0/). 